\title{
Energy harvesting with stacked dielectric elastomer transducers: Nonlinear theory, optimization, and linearized scaling law
}

\author{
A. Tutcuoglu ${ }^{1,2,3}$ and C. Majidi ${ }^{1}$ \\ ${ }^{1}$ Department of Mechanical Engineering, Carnegie Mellon University, Pittsburgh, Pennsylvania 15213, USA \\ ${ }^{2}$ Department of Aeronautics, Imperial College London, London SW7 2AZ, United Kingdom \\ ${ }^{3}$ Département de Mathématiques et Informatique, École Centrale de Lyon, 69134 Écully, France
}

(Received 22 November 2014; accepted 5 December 2014; published online 16 December 2014)

\begin{abstract}
Using principles of damped harmonic oscillation with continuous media, we examine electrostatic energy harvesting with a "soft-matter" array of dielectric elastomer (DE) transducers. The array is composed of infinitely thin and deformable electrodes separated by layers of insulating elastomer. During vibration, it deforms longitudinally, resulting in a change in the capacitance and electrical enthalpy of the charged electrodes. Depending on the phase of electrostatic loading, the DE array can function as either an actuator that amplifies small vibrations or a generator that converts these external excitations into electrical power. Both cases are addressed with a comprehensive theory that accounts for the influence of viscoelasticity, dielectric breakdown, and electromechanical coupling induced by Maxwell stress. In the case of a linearized Kelvin-Voigt model of the dielectric, we obtain a closed-form estimate for the electrical power output and a scaling law for DE generator design. For the complete nonlinear model, we obtain the optimal electrostatic voltage input for maximum electrical power output. (C) 2014 AIP Publishing LLC.
\end{abstract}

[http://dx.doi.org/10.1063/1.4904473]

Because they are soft and elastically deformable, dielectric elastomers (DEs) actuators and generators have the potential for integration in "smart" textiles, assistive wearable devices, or biologically inspired "soft robots" without introducing mechanical stiffness or kinematic constraints. When coated with fluidic or elastomeric conductors, a thin film of DE can function as an electrostatic actuator, ${ }^{1-5}$ capacitive sensor, ${ }^{6,7}$ or electrical generator. ${ }^{8-18}$ Here, we examine the special case of a "stacked capacitor" composed of an array of thin and deformable electrodes separated by layers of insulating elastomer, ${ }^{19,20}$ the former of which are subsequently modeled as infinitely thin. In contrast to existing designs that rely on rigid frames, the stacked capacitor contains no external kinematic constraints and hence exhibits the greatest coupling between changes in dielectric film thickness and capacitance. ${ }^{9}$ Moreover, with existing fabrication methods and materials, it can have virtually any size or prismatic geometry. Nonetheless, as with other DE transducer designs, the actuation or energy harvesting strongly depends on the timing of charging and discharging. ${ }^{21}$ When charging is in-phase with the forced harmonic oscillation, the vibration is damped and energy is extracted from the capacitor array in the form of electricity. For out-of-phase charging, the capacitors absorb electrostatic energy and the supplied charge induces Maxwell stresses that increase the amplitude of vibration.

A stacked DE transducer array is composed of alternating layers of conductive and insulating elastomer (Fig. 1(a)). Such structures have been produced by folding or bonding poly(dimethylsiloxane) (PDMS) film coated with carbon powder ${ }^{19}$ or a thin layer of conductive, carbon-filled PDMS (cPDMS). ${ }^{22}$ When placed on a harmonically vibrating structure, the elastic cylinder will deform in response to inertial and viscous forces as well as the so-called Maxwell stress exerted by electrostatic charge on the conductive elastomer electrodes. Referring to Fig. 1(b), the functions $u_{0}=u_{0}(t), y=y(t)$, and $u=u(z, t)$ represent the vertical displacement of the vibrating support, free end, and cross-

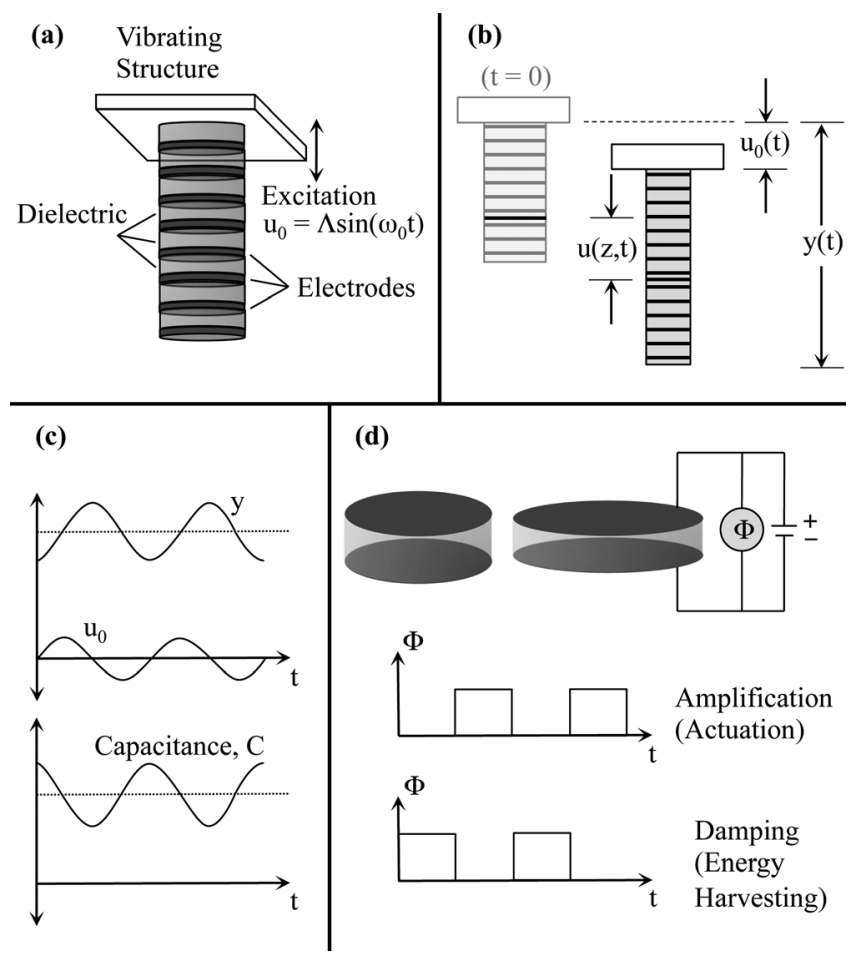

FIG. 1. (a) Illustration of DE transducer composed of alternating layers of insulating and dielectric elastomer. (b) Kinematics of deformation under harmonic excitation. (c) Change in tip position $(y(t))$, base position $\left(u_{0}(t)\right)$, and capacitance $(C)$. (d) Response of a capacitive element to applied voltage $\Phi$-applying voltage during compressive and tensile phases of oscillation will lead to amplification (actuation mode) and damping (energy harvesting), respectively. 
section with an initial distance $z$ from the support (in the natural state prior to vibration). For simplicity, it is assumed that $u_{0}$ has the form $\Lambda \sin \left(\omega_{0} t\right)$, where $\Lambda$ is the amplitude of the external excitation and $\omega_{0}$ is the excitation frequency. As shown in Figs. 1(c) and 1(d), such deformation leads to a harmonic change in capacitance that can be used to either amplify or damp motion when a voltage drop $\Phi=\Phi(t)$ is applied to the electrodes. These two operation modes correspond to mechanical actuation and electrical power generation, respectively. In both cases, response is strongly influenced by viscoelasticity, the timing and amplitude of applied voltage, dielectric breakdown, and nonlinear elasticity.

Here, we perform a numerical analysis and optimization to examine the influence of these effects on the electromechanical response of a stacked DE transducer. This model builds on previous studies on the mechanics of dielectric elastomer actuators (DEAs) ${ }^{10,23-27}$ and extends this existing work to determine the voltage $\Phi(t)$ that maximizes energy generation. Interestingly, we find that power output is maximized with a non-linear electric field input that deviates slightly from the square wave input shown in Fig. 1(d) and previously postulated in Ref. 21. Moreover, for large electric fields approaching the breakdown strength of the dielectric, Maxwell stress leads to a change in the resonant frequency at which electrical power output is maximized. This has important implications on the geometry of the DE transducer, which should be designed to have a resonant frequency at high fields that is close to the anticipated excitation frequency of the host structure. Lastly, using the closed-form solution to a linearized approximation of the governing field equation, we derive a scaling law that provides an order-of-magnitude estimate for the specific power $\mathscr{P}=\{$ power output $\} /$ volume $\}$. Although crude, this algebraic expression is useful for determining whether DE-based electrostatic energy harvesting is feasible for known excitation conditions.

The electrodes in the DE transducer stack are separated by an insulating elastomer (e.g., PDMS, acrylic-based VHB ${ }^{\circledR}$ tape, soft urethane) that can be modeled as an incompressible, isotropic, and viscoelastic solid. The electrodes themselves are assumed to remain conductive when deformed, have negligible elastic rigidity or viscosity, and have negligible volume compared to the surrounding elastomer. These assumptions allow the generator to be treated as a homogeneous solid governed by the mechanics of the dielectric. The vertical displacement $u=u(z, t)$ and longitudinal coordinate $z$ are defined for each cross-section of the prismatic solid with length $L$ and undeformed cross-section $A_{0}$ (Fig. 1(b)). A volumetric element $d V$ of the cylinder is subject to axial stresses $\left\{\sigma_{r r}, \sigma_{\theta \theta}, \sigma_{z z}\right\}$ in the radial $\left(\mathbf{e}_{r}\right)$, hoop $\left(\mathbf{e}_{\theta}\right)$, and longitudinal $\left(\mathbf{e}_{z}\right)$ directions. The elastomer is also subject to Maxwell stresses from electrostatic interactions between internal induced dipoles. These have a magnitude of $\sigma_{M}:=$ $(1 / 2) \epsilon E_{s}^{2}$ and exert tension on the $\mathbf{e}_{r}$ and $\mathbf{e}_{\theta}$ faces and compression on the $\mathbf{e}_{z}$ faces of $d V$. Here, $\epsilon=\epsilon_{r} \epsilon_{0}$ is the electric permittivity of the dielectric, $\epsilon_{r}$ is the relative permittivity, $\epsilon_{0}=8.85 \times 10^{-12} \mathrm{~F} / \mathrm{m}$ is the vacuum permittivity. The internal electric field $E_{s}=E_{s}(z, t)=\Phi / d$ corresponds to the voltage drop $\Phi(t)$ applied across the thickness $d(z, t)$ of the deformed dielectric layer.
It is assumed that the dielectric is a Neo-Hookean solid with a coefficient of elasticity $C_{1}$ and elastic strain energy density $W=C_{1}\left(\lambda_{r}^{2}+\lambda_{\theta}^{2}+\lambda_{z}^{2}-3\right)$. Here, $\lambda_{r}$ and $\lambda_{\theta}$ are the stretches in the radial and hoop directions, and $\lambda_{z}=$ $1+\partial u / \partial z$ is the stretch in the longitudinal direction. Moreover, a Kelvin-Voigt model with a viscosity $\eta$ is used to account for viscoelasticity in the longitudinal direction. Lastly, each volumetric element is subject to a hydrostatic pressure $p$ that is required to maintain incompressibility, i.e., $J:=\lambda_{r} \lambda_{\theta} \lambda_{z}=1$. The combination of mechanical tractions, electrostatic Maxwell stress, and hydrostatic pressure result in the following constitutive relationships:

$$
\begin{gathered}
\sigma_{r r}+\sigma_{M}=\lambda_{r} \frac{\partial W}{\partial \lambda_{r}}-p, \\
\sigma_{\theta \theta}+\sigma_{M}=\lambda_{\theta} \frac{\partial W}{\partial \lambda_{\theta}}-p, \\
\sigma_{z z}-\sigma_{M}=\lambda_{z} \frac{\partial W}{\partial \lambda_{r}}-p+\eta \frac{\partial \lambda_{z}}{\partial t} .
\end{gathered}
$$

Since the surface of the cylinder is traction free, it follows that $\sigma_{r r}=\sigma_{\theta \theta}=0$. Noting that $J=1$, this implies that $\lambda_{r}=\lambda_{\theta}=\lambda_{z}^{-1 / 2}$. From the constitutive relationships, it follows that the dielectric must have a hydrostatic pressure $p=2 C_{1} \lambda_{z}^{-1}-(1 / 2) \epsilon E_{s}^{2}$. Therefore,

$$
\sigma_{z z}=2 C_{1}\left\{\lambda_{z}^{2}-\frac{1}{\lambda_{z}}\right\}+\epsilon E_{s}^{2}+\eta \frac{\partial \lambda_{z}}{\partial t} .
$$

Performing the linear momentum balance on $d V$ in the $\mathbf{e}_{z}$ direction implies $\partial \sigma_{z z} / \partial z=\rho\left\{\partial^{2} u / \partial t^{2}\right\}$. Substituting (4) and $\lambda_{z}=1+\partial u / \partial z$ into this yields a governing field equation of the form

$$
\begin{gathered}
2 C_{1} \frac{\partial^{2} u}{\partial z^{2}}\left\{2\left(1+\frac{\partial u}{\partial z}\right)+\left(1+\frac{\partial u}{\partial z}\right)^{-2}\right\} \\
+2 \epsilon E_{s} \frac{\partial E_{s}}{\partial z}+\eta \frac{\partial^{3} u}{\partial z^{2} \partial t}=\rho \frac{\partial^{2} u}{\partial t^{2}} .
\end{gathered}
$$

The solution to this governing equation must satisfy the following kinematic and static boundary conditions:

$$
\begin{array}{ll}
u=\Lambda \sin \left(\omega_{0} t\right) & z=0, \forall t>0 \\
\sigma_{z z}=0 & z=L, \forall t>0 \\
u=f(z), \quad \text { where } f(L)=0 & t=0, \forall z \in[0, L] \\
u_{, t}=g(z), \quad \text { where } g(L)=0 & t=0, \forall z \in[0, L] .
\end{array}
$$

The functions $f(z)$ and $g(z)$ are the prescribed initial vertical displacement and velocity of the cross-sectional elements.

At any given instant in time, the power output $P_{\text {out }}=P_{\text {out }}(t)$ is calculated as the product of $\Phi(t)$ and the total current of electricity $I(t)$ from the electrodes to the power supply. For a large number of electrodes, the capacitors may be treated as a continuum of infinitesimal cross-sectional elements with area $A$ and thickness $\delta$. This corresponds to a capacitance $d C=\epsilon A / \delta$, voltage drop $d V=E_{s} \delta$, and current $d I=\partial / \partial t\{d C d V\}$. The instantaneous power output is then estimated by integrating $d I d V$ over $z \in[0, L]$ : 


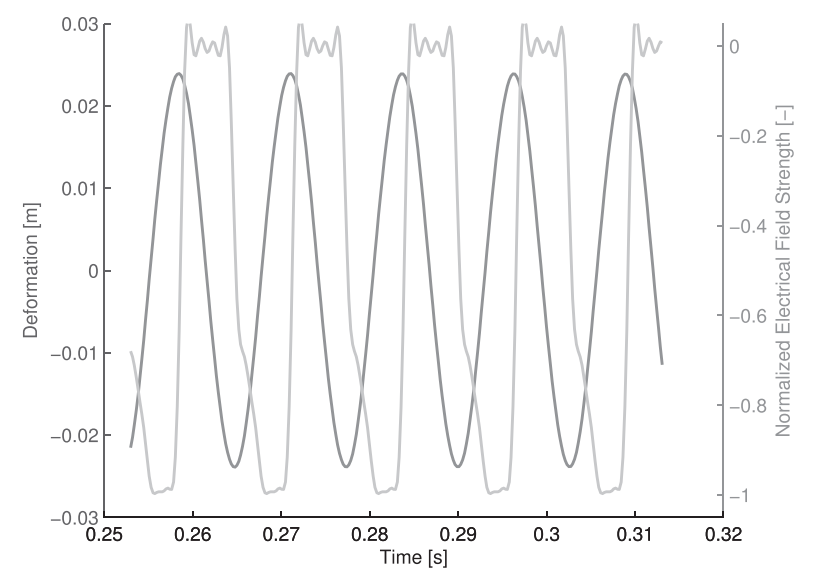

FIG. 2. Optimal electric field for maximizing electrical power output during forced vibration.

$$
P_{\text {out }}=\int_{z=0}^{z=L}-\frac{\epsilon E_{0} A_{0}}{\lambda_{z}^{2}}\left\{\frac{\partial E_{0}}{\partial t}-\frac{2 E_{0}}{\lambda_{z}} \frac{\partial \lambda_{z}}{\partial t}\right\} d z
$$

Instead of prescribing $E_{s}$ (which requires a priori knowledge of the stretch $\lambda_{z}$ ), we instead prescribe the "nominal" electric field $E_{0}=\Phi / d_{0}$, where $d_{0}$ is the natural thickness of each dielectric layer. Energy harvesting optimization is performed by parameterizing $E_{0}$ with a Fourier series of order $N$

$$
E_{0}(t)=\frac{a_{0}}{2}+\sum_{i=1}^{N}\left\{a_{i} \cos \left(i \omega_{0} t\right)+b_{i} \sin \left(i \omega_{0} t\right)\right\},
$$

where $a_{0}$ and $a_{i}, b_{i}, i \in\{1, \ldots, N\}$ are treated as the control variables. An optimization is performed to determine the control variables that maximize the average value $\left(P_{\text {avg }}\right)$ of $P_{\text {out }}$ over a single oscillatory cycle with period $T=2 \pi / \omega_{0}$.

The results presented in Figs. 2 and 3 are obtained with the following set of parameters: $L=0.1 \mathrm{~m}, A_{0}=0.001 \mathrm{~m}^{2}$, $C_{1}=0.167 \mathrm{MPa}, \eta=100 \mathrm{~Pa}-\mathrm{s}, \quad \rho=1000 \mathrm{~kg} / \mathrm{m}^{3}, \quad \epsilon_{r}=1$. It should be noted that for most dielectrics, $\epsilon_{r}$ is typically in the range of 2-5. However, we have selected $\epsilon_{r}=1$ as a general order-of-magnitude estimate since the exact value will be material dependent. Solutions for $u=u(z, t)$ were obtained in MATLAB 2013a (The Mathworks, Inc.) using the pdepe

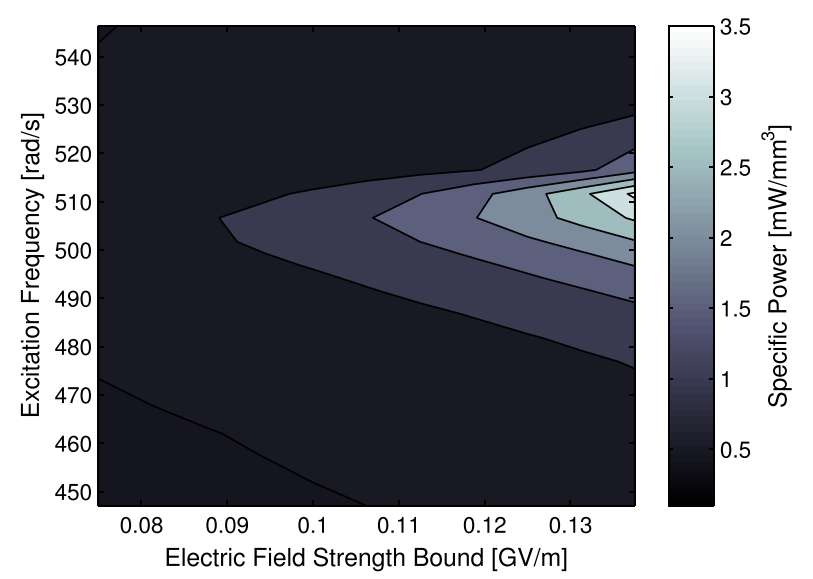

FIG. 3. Specific Work Output $\mathrm{P}_{\text {out }}$ as function of base- and electric excitation frequency and electric field strength bound taking into account Maxwell stress at optimal phase excitation. parabolic PDE solver for 200 spatial elements, 1500 time steps, and a simulation time of $25 \times \mathrm{T}$. Optimization was performed with optimtool to obtain Fourier coefficients for the optimal field $E_{0}=E_{0}(t)$. The objective function in this optimization procedure is the supplied electrostatic energy over five steady-state oscillatory cycles.

The optimal field for maximizing average power output is presented in Fig. 2. This result is consistent with the postulate that DE energy harvesting is optimized with a squarewave for applied voltage. ${ }^{21}$ However, close inspection shows slight deviation at the end of each period. This arises from the small but noticeable influence of longitudinal stretch $\lambda_{z}$ on the internal electric field. If we divide $E_{S}$ by the minimum stretch $\lambda_{\text {min }}(t)=\min _{z \in[0, L]} \lambda_{z}(z, t)$, then a square wave is recovered. Thus, it can be concluded that previous postulates regarding optimal control are approximately correct but not exact. They are only exact when the electric field is small and the influence of Maxwell stresses on mechanical deformation be neglected.

Referring to Fig. 3, we also observe that for higher fields, the specific power $\mathscr{P}=P_{\text {avg }} /\{$ volume $\}$ is maximized for excitation frequencies $\omega_{0}$ that exceed the natural $\omega_{n}$ frequency. This is attributed to out-of-phase deformation of individual capacitive elements and the loss of synchrony with applied voltage. For a prescribed maximum applied field $\Omega$, the specific power output can be maximized by selecting an optimal frequency and phase for the applied electric field.

Finally, an approximate estimate for the electrical power output is obtained by assuming a Hookean Kelvin-Voigt solid: $\sigma_{z z}=E\left(\lambda_{z}-1\right)+\eta\left\{\partial \lambda_{z} / \partial t\right\}$, where $E$ is Young's modulus and the contribution of Maxwell stress to $u$ is neglected. In this case, the governing PDE reduces to $E\left\{\partial^{2} u / \partial z^{2}\right\}+\eta\left\{\partial^{3} u / \partial z^{2} t\right\}=\rho\left\{\partial^{2} u / \partial t^{2}\right\}$. A closed form solution for $u=u(z, t)$ is presented in the Supplementary material. $^{28}$ The longitudinal stretch $\lambda_{z}$ is approximated as $1+u_{L} / L$, where $u_{L}=-X \cos \left(\omega_{n} t\right)$ and $X$ is the amplitude of the displacement of the free end at steady state. The instantaneous power output $P_{\text {out }}$ is estimated for a prescribed electric field electric field $E_{s}=(\Omega / 2)\left\{1+\sin \left(\omega_{n} t\right)\right\}$. The amplitude, $\Omega$ represents the maximum allowable applied electric field, which is bounded above by the dielectric breakdown strength $\left(E_{b}\right)$ of the elastomer. As before, the instantaneous power output is then estimated by integrating $d I d V$ over $z \in[0, L]$. This yields the following approximation:

$$
\begin{aligned}
P_{\text {out }}(t)= & -\frac{1}{2} A_{0} \omega_{n} \epsilon \Omega^{2} \cos \left(\omega_{n} t\right)\left\{1+\sin \left(\omega_{n} t\right)\right\} \\
& \times\left\{L-X \cos \left(\omega_{n} t\right)\right\},
\end{aligned}
$$

The average specific power output $\mathscr{P}$ is obtained by integrating $P_{\text {out }} / T$ over $t \in[0, T]$, where $T=2 \pi / \omega_{n}$ is the period of oscillation and dividing by the total volume $A_{0} L$

$$
\mathscr{P}=\frac{\epsilon \Omega^{2} \omega_{n} X}{8 L} .
$$

This is a crude approximation since it ignores the incompressibility and nonlinear elasticity of the dielectric or the influence of Maxwell stress on the amplitude $X$. Moreover, direct 
comparison with the results in Fig. 3 are not possible since the strains there are large and the Maxwell stresses associated with an applied field of $\sim 10^{8} \mathrm{Vm}^{-1}$ are too large to ignore. Nonetheless, (10) provides a reliable order-of-magnitude estimate for lower applied fields and oscillatory amplitudes. In general, for a permittivity $\sim 1$ and resonant frequency on the order of $0.01-1 \mathrm{kHz}$, the specific power output is expected to range form 0.01 to $1 \mathrm{~mW} / \mathrm{mm}^{3}$. This is comparable to other modes of vibrational energy harvesting $^{9,21,29,30}$ and suggests that DE generators have the potential to power personal electronics and other body-mounted technologies.

In summary, this work examines the mechanics of deformation of a multi-layer DE generator and identifies the optimal electrostatic loading $E_{0}(t)$ that maximizes average power output $P_{\text {avg }}$. Using a linearized theory, we obtain a scaling law $\mathscr{P} \approx P_{\text {avg }} /\{$ volume $\}$ that suggests that $\mathrm{DE}$ generators have potentially similar performance to other methods of vibrational energy harvesting. For large deformation and electric fields, we must use a nonlinear analysis that accounts for Maxwell stress and treats the elastomer as a NeoHookean solid. Parameterizing $E_{0}$ with a Fourier series, we determine that the electrodes should be charged with a square-wave voltage unless excited at near-breakthrough field strengths, in which case a modified square wave has to be applied. The amplitude $\Omega$ of the applied electric field also influences the excitation frequency at which the electrical power output can be optimized.

This work was supported by the Air Force Office of Scientific Research (AFOSR) Young Investigator Program (Mechanics of Multifunctional Materials and Microsystems; Program Manager: Les Lee).

${ }^{1}$ O. A. Araromi, I. Gavrilovich, J. Shintake, S. Rosset, M. Richard, V. Gass, and H. R. Shea, IEEE/ASME Trans. Mechatronics 20, 438 (2015).

${ }^{2}$ O. A. Araromi, I. Gavrilovich, J. Shintake, S. Rosset, and H. Shea, Proc. SPIE 9056, 90562G (2014).

${ }^{3}$ P. Brochu and Q. Pei, Macromol. Rapid Commun. 31, 10 (2010).
${ }^{4}$ F. Carpi and E. Smela, Biomedical Applications of Electroactive Polymer Actuators (Wiley Online Library, 2009).

${ }^{5}$ F. Carpi, D. De Rossi, R. Kornbluh, R. E. Pelrine, and P. Sommer-Larsen, Dielectric Elastomers as Electromechanical Transducers (Elsevier, 2011).

${ }^{6}$ R. D. Kornbluh, R. Pelrine, Q. Pei, R. Heydt, S. Stanford, S. Oh, and J. Eckerle, Proc. SPIE 4698, 254-270 (2002).

${ }^{7}$ S. Rosset and H. Shea, Appl. Phys. A 110, 281 (2013).

${ }^{8}$ B. Czech, R. van Kessel, P. Bauer, J. Ferreira, and A. Wattez, in 14th International Power Electronics and Motion Control Conference (EPE/ PEMC) (IEEE, 2010), pp. S4-18-S4-23.

${ }^{9}$ S. Jin Adrian Koh, C. Keplinger, T. Li, S. Bauer, and Z. Suo, IEEE/ASME Trans. Mechatronics 16, 33 (2011).

${ }^{10}$ T. Li, S. Qu, and W. Yang, J. Appl. Phys. 112, 034119 (2012).

${ }^{11}$ A. O'Halloran, F. O'Malley, and P. McHugh, J. Appl. Phys. 104, 071101 (2008).

${ }^{12}$ I. A. Anderson, E. P. Calius, T. Gisby, T. Hale, T. McKay, B. O’Brien, and S. Walbran, Proc. SPIE 7287, 72871H (2009).

${ }^{13}$ I. Anderson, T. Hale, T. Gisby, T. Inamura, T. McKay, B. O'Brien, S. Walbran, and E. Calius, Appl. Phys. A 98, 75 (2010).

${ }^{14}$ I. A. Anderson, T. C. H. Tse, T. Inamura, B. M. O'Brien, T. McKay, and T. Gisby, Appl. Phys. Lett. 98, 123704 (2011).

${ }^{15}$ I. A. Anderson, T. A. Gisby, T. G. McKay, B. M. O’Brien, and E. P. Calius, J. Appl. Phys. 112, 041101 (2012).

${ }^{16} \mathrm{R}$. Kornbluh, R. Pelrine, J. Eckerle, and J. Joseph, in Proceedings of the 1998 IEEE International Conference on Robotics and Automation (IEEE, 1998), Vol. 3, pp. 2147-2154.

${ }^{17}$ J. Zhu, S. Cai, and Z. Suo, Polym. Int. 59, 378 (2010).

${ }^{18}$ J. Zhu, S. Cai, and Z. Suo, Int. J. Solids Struct. 47, 3254 (2010).

${ }^{19}$ G. Kovacs, L. Düring, S. Michel, and G. Terrasi, Sens. Actuators, A 155, 299 (2009).

${ }^{20}$ G. Kovacs and L. Düring, Proc. SPIE 7287, 72870A (2009).

${ }^{21}$ B. C. Yen and J. H. Lang, IEEE Trans. Circuits Syst. I 53, 288 (2006).

${ }^{22}$ F. Carpi, C. Salaris, and D. D. Rossi, Smart Mater. Struct. 16, S300 (2007).

${ }^{23}$ J. Fox and N. Goulbourne, J. Mech. Phys. Solids 56, 2669 (2008).

${ }^{24}$ D. L. Henann, S. A. Chester, and K. Bertoldi, J. Mech. Phys. Solids 61, 2047 (2013).

${ }^{25}$ W. Hong, J. Mech. Phys. Solids 59, 637 (2011).

${ }^{26} \mathrm{~T}$. Li, C. Keplinger, R. Baumgartner, S. Bauer, W. Yang, and Z. Suo, J. Mech. Phys. Solids 61, 611 (2013).

${ }^{27}$ X. Zhao and Z. Suo, Appl. Phys. Lett. 91, 061921 (2007).

${ }^{28}$ See supplementary material at http://dx.doi.org/10.1063/1.4904473 for a solution to the linearized model.

${ }^{29}$ K. A. Cook-Chennault, N. Thambi, and A. M. Sastry, Smart Mater. Struct. 17, 043001 (2008).

${ }^{30}$ R. Kaltseis, C. Keplinger, R. Baumgartner, M. Kaltenbrunner, T. Li, P. Mchler, R. Schwdiauer, Z. Suo, and S. Bauer, Appl. Phys. Lett. 99, 162904 (2011). 